\title{
Towards an immunoscore for triple negative breast cancer (TNBC): lymphocytic infiltrate predicts outcome
}

\author{
Sylvia Adams ${ }^{1 *}$, Robert Gray ${ }^{1}$, Sandra Demaria', Lori J Goldstein', Edith A Perez², Lawrence N Shulman³, \\ Silvana Martino ${ }^{4}$, Nancy E Davidson'1, George W Sledge', Joseph A Sparano', Sunil Badve ${ }^{1}$ \\ From Society for Immunotherapy of Cancer 28th Annual Meeting \\ National Harbor, MD, USA. 8-10 November 2013
}

\section{Background}

Efforts are ongoing to further classify solid tumors by immunoscores, which have prognostic implications and may also have predictive value and identify targets for novel therapies to increase cure rates. TNBC are highly aggressive breast cancers, which are frequently infiltrated by lymphocytes, recently linked to prognosis in lymph node (LN)-positive TNBC.

\section{Methods}

Full face hematoxylin \& eosin (H\&E)-stained tumor sections of 500 randomly selected TNBC (LN positive and negative) from two adjuvant US clinical trials (E1199, E2197) were assessed for density and location of TILs. Associations with outcomes after adjuvant chemotherapy were determined and models were performed to identify relationships with established prognostic factors.

\section{Results}

481 tumors were evaluable, stromal tumor infiltrating lymphocytes (sTIL) were observed in $80 \%$ of cancers; $49 \%$, $27 \%$ and $4 \%$ of tumors had a $10 \%, 20-40 \%$ and $50 \%$ or greater infiltrate, respectively. With a median follow-up of 10.6 years the degree of sTIL was positively correlated with disease-free survival (DFS), distant recurrence-free interval and overall survival. There were associations between sTIL scores and LN status but not age, menopausal status or tumor size. The likelihood of a sTIL score of 0 decreased as the number of positive LN increased, with a rate of $25.4 \%$ (50/197) for LN negative breast cancers, $18.1 \%(36 / 50)$ for tumors with $1-3$ positive LN and $10.6 \%$
$(9 / 85)$ for tumors with $>3 \mathrm{LN}(\mathrm{P}=0.01)$. For DFS, the estimated HR was $0.84(0.74,0.95, \mathrm{P}=0.005)$ for a 10 point change in STIL, which increased further in significance when $\mathrm{LN}$ involvement ( 0 vs $1-3$ vs $>3$ ) was taken into account (HR 0.82; 0.72,0.94, $\mathrm{P}=0.003$ ).

\section{Conclusion}

We demonstrated that sTIL can improve outcome prediction in TNBC beyond traditional TNM staging. Our data provide clinical validation of recent findings by Loi et al, JCO 2013 using identical methods. We therefore propose that evaluation of stromal lymphocytic infiltrates in H\&E-stained tumor sections provides a simple method to establish an immunoscore for this subtype of breast cancer.

\section{Authors' details}

${ }^{1}$ Eastern Cooperative Oncology Group, Philadelphia, PA, USA. ${ }^{2}$ North Central Cancer Treatment Group, Rochester, MN, USA. ${ }^{3}$ Cancer and Leukemia Group B, Chicago, IL, USA. ${ }^{4}$ Southwest Oncology Group, Portland, OR, USA.

Published: 7 November 2013

doi:10.1186/2051-1426-1-S1-022

Cite this article as: Adams et al:: Towards an immunoscore for triple negative breast cancer (TNBC): lymphocytic infiltrate predicts outcome. Journal for ImmunoTherapy of Cancer 2013 1(Suppl 1):O22.

${ }^{1}$ Eastern Cooperative Oncology Group, Philadelphia, PA, USA

Full list of author information is available at the end of the article

(c) 2013 Adams et al; licensee BioMed Central Ltd. This is an Open Access article distributed under the terms of the Creative Commons Attribution License (http://creativecommons.org/licenses/by/2.0), which permits unrestricted use, distribution, and reproduction in any medium, provided the original work is properly cited. 